\title{
Base dependent 1,3-dithioacetals rearrangement over sulfoxidation under visible-light photocatalysis to access disulfide- linked-dithioesters
}

\author{
Pankaj D. Dharpure ${ }^{\mathrm{a}}$, Mousumi Behera ${ }^{\mathrm{a}}$, Archana S. Thube ${ }^{\mathrm{a}}$ and Ramakrishna G. Bhat ${ }^{\mathrm{a} *}$ \\ ${ }^{a}$ Department of Chemistry, Indian Institute of Science Education and Research (IISER)-Pune, Dr. \\ Homi Bhabha Road, Pashan, 411008, Pune, Maharashtra, India.
}

\begin{abstract}
Base dependent oxidative rearrangement of dithiolanes and dithianes to access disulfide-linkeddithioesters under visible-light photoredox catalytic conditions has been disclosed. The protocol demonstrated the ability to synthesize either rearranged product or sulfoxide by simply switching the base with inherent ability to make hydrogen bonding with sulfur atom. Unlike, the usual deprotection of thioacetals to corresponding aldehydes under the oxidative conditions, we observed the unique regioselective oxidative reactivity of thioacetals to form disulfide-linked-dithioester or sulfoxides. The generality of the protocol has been demonstrated by exploring a wide range of substrates. As an application the in-situ generated thyil radical has been trapped with disulfides to prepare hetrodisulfides of potential utility. The protocol proved to be practical on gram scale quantity and relied on clean energy source for the transformation. Based on the series of control experiments, cyclic volametry and Stern-Volmer studies the plausible mechanism has been proposed.
\end{abstract}

\section{Introduction}

The rearrangement reactions in organic chemistry offer an unconventional route to access a wide variety of molecular scaffolds in one pot. It is well known in the literature that many of the sulfur compounds are known to undergo rearrangements under Lewis as well as Brønsted acid conditions. ${ }^{1}$ The cyclic thioacetals are very useful intermediates in organic synthesis and have been well explored for the umpolung reactivity in a variety of transformations. ${ }^{2}$ Interestingly, ability of cyclic thioacetals for the rearrangement reactions have been rarely explored in the literature to generate the thioesters. The molecules containing thioester moiety are one of the few important intermediates in biosynthetic pathways such as fatty acid, esters, polyketide, non-ribosomal peptide synthesis. ${ }^{3}$ Also, thioester moiety plays a vital role in the native chemical ligation by S-N shift, lipidation of G-coupled protein for the signal regulation and also it acts as probe for the enzymatic assembly lines. ${ }^{4}$ These useful compounds have been utilized in the solid phase synthesis of phosphorodithioate-modified DNA that has a wide utility for the gene silencing technology. ${ }^{5}$ Traditionally, the thioesters are synthesized from the reaction of carboxylic acids, acid chloride or acid anhydride and thiols. ${ }^{6}$ Thioesters have also been achieved using metal catalyst by thiocarbonylation. ${ }^{7}$ However, along these protocols many other reported methods are not clean and require the use of metal catalysts, toxic reagents and activating-promoting reagents. ${ }^{8}$ Similarly, disulfide-linkage plays an important role in maintaining the structure, function and stability of proteins and peptides because of its reversible-nature in the biological condition. ${ }^{9}$

Undoubtedly, due to the reactivity and several use of thioesters and disulfides, these scaffolds have been widely utilized for labelling of the biomolecules (proteins, nucleic acids, carbohydrates, lipids).$^{10}$ Also these scaffolds are extensively used in the drug delivery applications as well as synthesis of biomaterials. ${ }^{11}$ Despite the enormous utility of both disulfides and thioesters, there are not many straightforward syntheses to prepare the molecule containing both disulfide and thioester moieties. Few available methods require multi-steps to synthesize the same. ${ }^{5}$

Scheme 1. Reactivity of Thioacetals in organic synthesis

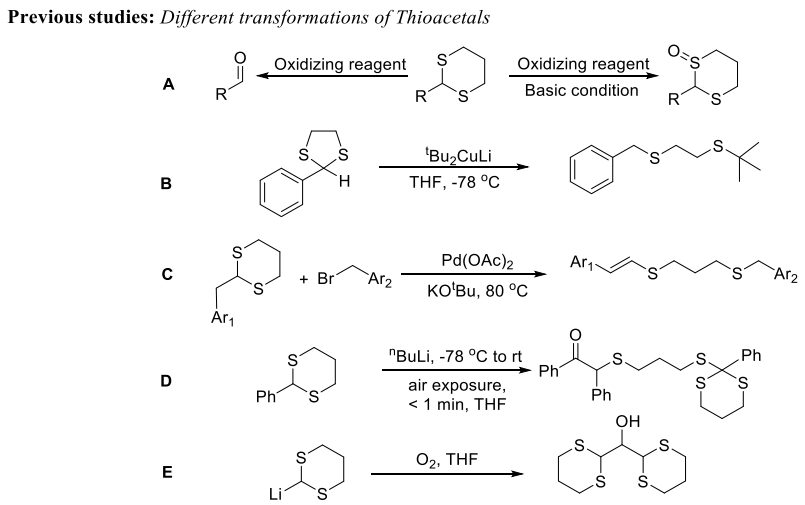

Present study: Visible light mediated base selective rearrangement and sulfoxidation of dithioacetals F.

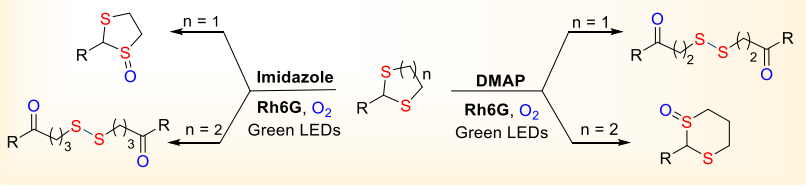

The photoredox catalysis is an emerging research area in organic synthesis that provides a milder and sustainable approach to carry out the useful organic transformations using visible light. ${ }^{12}$ Many molecules are known to show interesting reactivities under photocatalysis. The cleavage of C-S bond has been well studied under the photoredox condition in the presence of oxidizing reagent. ${ }^{13}$ However, surprisingly, the selective cleavage of single $\mathrm{C}-\mathrm{S}$ bond of cyclic thioacetal under the oxidizing reaction condition has not been reported till date 
to the best of our knowledge. This may be presumably due to the difficulty in controlling the selective cleavage of one of the two C-S bonds of thioacetal under oxidizing conditions. ${ }^{14}$ Generally, cleavage of first C-S bond would simultaneously lead to the breaking of the second C-S bond to furnish the corresponding deprotected compounds (aldehydes) or the oxidation of sulfur to generate the sulfoxide. ${ }^{14,15}$ Alternatively, researchers have explored different transformations by exploring the potential utility of in-situ generated lithiatedthioacetals that is known to proceed through the cleavage of single C-S bond of thioacetals. ${ }^{16,17}$ However, these protocols require low temperature and strong base such as $n$-butyl lithium. Schmink and coworkers demonstrated synthesis of dithioether via the selective cleavage of C-S bond of thioacetals in the absence of oxidizing reagent however at an elevated temperature using metal catalyst $\operatorname{Pd}(\mathrm{OAc})_{2 .}{ }^{18}$ However this protocol was limited to thioacetals containing benzylic $\beta$-hydrogen to sulfur atom of thioacetals. The efforts to cleave the single $\mathrm{C}-\mathrm{S}$ bond of thioacetal selectively have led to interesting and different products (Scheme 1). However, it is highly challenging and demanding to execute selective cleavage of single C-S bond of thioacetal under oxidizing condition to access the thioesters under metal free milder condition. Our research group has been working on photoredox catalysis and while we were exploring the deprotection of thioacetals under visible light photoredox catalysis to a corresponding aldehyde ${ }^{14 a}$ we presumed that possibly the deprotection is going through a couple of reactive intermediates based on the literature report of Peñéñory et al. ${ }^{14 \mathrm{~b}}$ We hypothesized that the choice of suitable bases under appropriate photoredox conditions may lead to the formation of thioesters. ${ }^{19}$ Herein, we present the one pot selective synthesis of disulfide-linked-dithioester or sulfoxides by simply switching the bases using photoreodox catalysis under oxygen atmosphere at room temperature starting from 1,3dithiolanes/1,3-dithianes. The transformation involves the rearrangement of cyclic thioacetals in presence of photocatalyst, mild base under oxygen atmosphere through single C-S bond cleavage using the visible light irradiation.

\section{Results and Discussion}

In order to validate the hypothesis, we commenced with a model reaction of $1 \mathbf{a}$ in presence of Eosin $\mathrm{Y}$ and $\mathrm{K}_{2} \mathrm{CO}_{3}$ under green LEDs $(15 \mathrm{~W})$ at room temperature and open-air condition to afford the unanticipated product disulfide-linkeddithioester $\mathbf{2 a}$ along with deprotection product 1a' (Table 1, entry 1). ${ }^{19}$ While the reaction in presence of Rhodamine $6 \mathrm{G}$ (Rh6G) under the reaction conditions furnished the similar results as observed in case of Eosin Y (Table 1, entry 2). Interestingly, the reaction of $\mathbf{1 a}$ in presence of $\mathrm{Rh} 6 \mathrm{G}, \mathrm{K}_{2} \mathrm{CO}_{3}$ in presence of oxygen atmosphere under green LEDs (15 W) at room temperature afforded the corresponding disulfidelinked-dithioester $\mathbf{2 a}$ in relatively higher yields (60\%, Table 1 , entry 3$)$. In order to optimize the reaction further we screened different photocatalysts ranging from Eosin Y, Rh6G, $\mathrm{Ru}(\mathrm{bpy})_{3} \mathrm{Cl}_{2}, \operatorname{Ir}(\mathrm{bpy})_{3}$, Acridinium-perchlorate and Pyrilliumtetrafluroborate. Among all we observed that Rh6G is more suitable for the desired transformation (ESI-2.1). Anhydrous acetonitrile proved to be more advantageous for the desired transformation based on the solvent screening (ESI-2.2). We also observed that for the desired transformation base, oxygen atmosphere, catalyst (Rh6G) and green LEDs are absolutely essential (Table 1, entries 4-7). Even at elevated reaction temperature $\left(60{ }^{\circ} \mathrm{C}\right)$ reaction did not work indicating the necessity of visible light (Table 1, entry 7).

Table 1. Optimization of the reaction

En
try

${ }^{\mathrm{a}}$ General reaction conditions: 1a $(0.2 \mathrm{mmol}), \mathrm{K}_{2} \mathrm{CO}_{3}(0.4 \mathrm{mmol})$, DMAP $(0.4$ $\mathrm{mmol})$, Imd (1 mmol), ACN (2 mL), catalyst (2mol\%), Green LEDs (15 W),

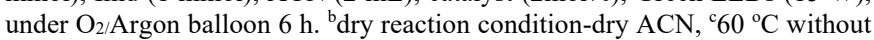
light source, dry acetonitrile $1.8 \mathrm{~mL}$, dry toluene $0.2 \mathrm{~mL}$, ${ }^{\mathrm{e}}$ dry $\mathrm{ACN}$, ${ }^{\mathrm{f}} \mathrm{dry}$ acetonitrile $1.5 \mathrm{~mL}$, dry $t$-butanol $0.5 \mathrm{~mL}$, Yields are in percentage

In order to optimize the reaction further we screened the reaction of $\mathbf{1 a}$ in presence of DMAP as a base while maintaining other optimized conditions, interestingly we observed that desired product $\mathbf{2 a}$ was formed in good yield (70\%, Table 1, entry 8 and ESI 2.3 ) and the corresponding sulfoxide 3a was formed in trace amount. Surprisingly, the reaction of 1a in presence of imidazole in anhydrous acetonitrile furnished the corresponding sulfoxide $\mathbf{3 a}$ in higher yield while the yield of disulfide-linked-dithioester $\mathbf{2 a}$ decreased significantly (Table 1, entry 9). We also observed that the increase in the yield of 3a when the reaction was performed in the mixture of anhydrous acetonitrile and $t$ butanol as reaction solvent (ESI 2.5). Our further attempts to optimize the reaction using other additives and the oxidizing reagents did not prove to be beneficial (ESI-2.4). Based on exhaustive screening, 2-(4-methoxyphenyl)-1,3-dithiolane 1a, DMAP, Rh6G ( $2 \mathrm{~mol} \%$ ) in anhydrous solvent (ACN: toluene; $9: 1, \mathrm{v} / \mathrm{v}$ ) under oxygen atmosphere proved to be the optimized reaction condition to obtain rearrangement product: disulfidelinked-dithioester 2a. Similarly, 2-(4-methoxyphenyl)-1,3dithiolane 1a, imidazole, Rh6G (2 mol\%) in anhydrous solvent $\left(\mathrm{ACN}:{ }^{t} \mathrm{BuOH} ; 3: 1, \mathrm{v} / \mathrm{v}\right)$ under oxygen atmosphere proved to be optimum reaction condition to obtain $\mathbf{3 a}$ exclusively.

Having obtained the optimized reaction conditions for both the transformations, we further planned to explore the substrate scope for the rearrangement as well as sulfoxidation reactions for the practicality and generality of the protocols. In this regard, the reactions of different dithiolanes 1 were explored 
under two different optimized reaction conditions to obtain the corresponding products (2a-2z, 2aa-2ac) and 3a-3d selectively (Scheme 2). We observed that different dithiolanes containing electron donating as well as electron withdrawing substituents at $p$-position afforded the corresponding rearranged products ( $\mathbf{2 a - 2 g )}$ in very good yields (up to $82 \%$, Table 2 ). We also observed that $m$-substituted ditholanes also offered the corresponding products $(\mathbf{2 h}, \mathbf{2 i}, \mathbf{2 k})$ in good yields (up to $71 \%$, Scheme 2).

Scheme 2. Substrate scope for rearrangement and sulfoxidation of dithiolane ${ }^{\mathrm{a}}$

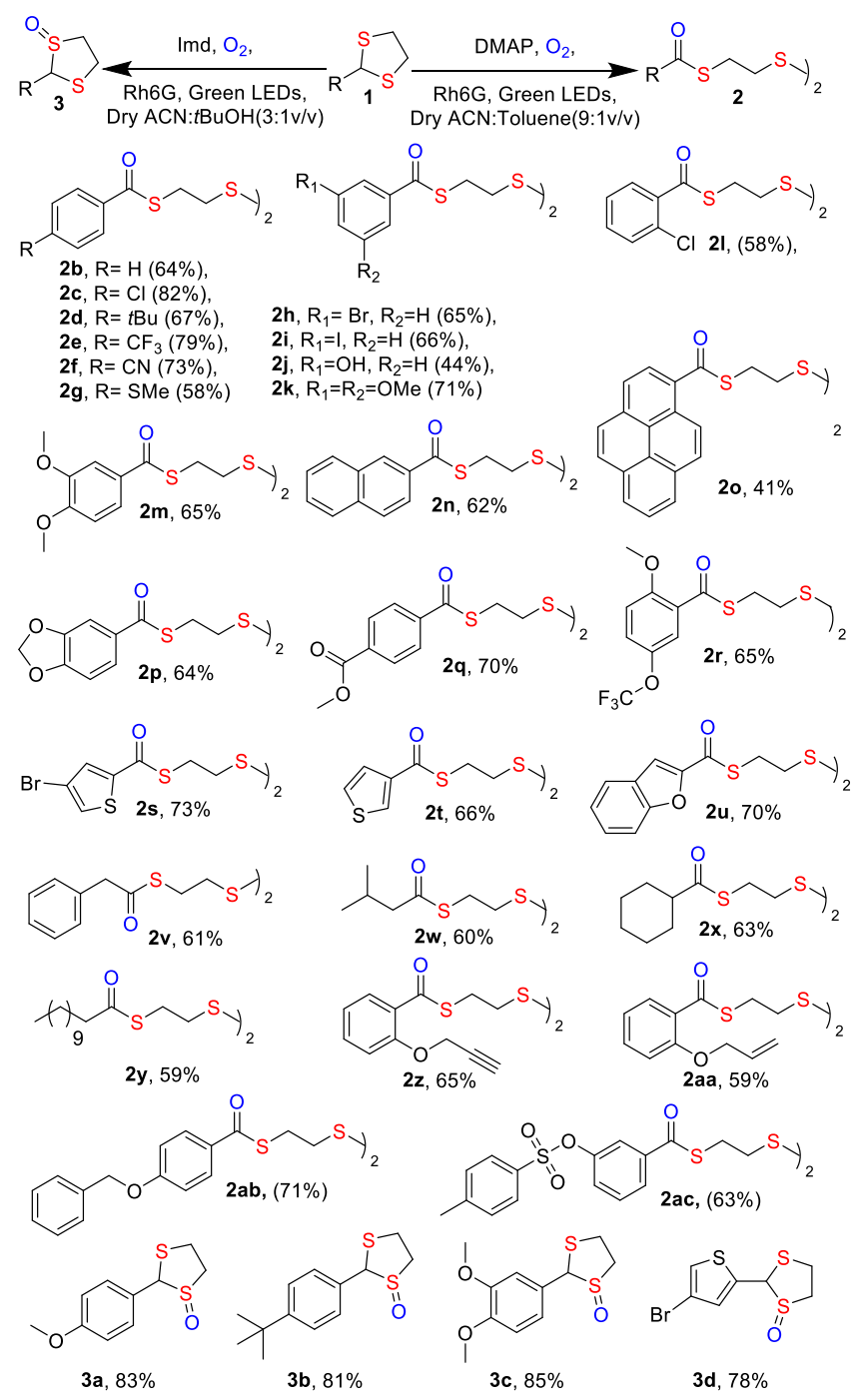

${ }^{\mathrm{a}}$ General reaction conditions: $1(0.2 \mathrm{mmol})$, DMAP $(0.4 \mathrm{mmol})$, Imidazole (1 $\mathrm{mmol}$ ), Solvent (2 ml), Green LEDs (15 W), under $\mathrm{O}_{2}, 6-12 \mathrm{~h}$.

However, the reaction of dithiolanes containing free hydroxyl and chloro substituents at $o$-position furnished the products $(\mathbf{2} \mathbf{j}$, 2l) in poor yields. While, the polyaromatic derivatives as well as dioxalane substituted dithiolanes under the optimized reaction conditions gave the corresponding products $(\mathbf{2 n - 2 p})$ in good yields (Scheme 2). The dithiolanes containing ester as well as ether moiety under the reaction condition afforded the products $(\mathbf{2 q}, \mathbf{2 m}, \mathbf{2 r})$ in good yields.
Even, the different heterocyclic and aliphatic dithiolanes worked smoothly under reaction conditions to furnish the rearranged products (2s-2y) in good yields. In order to explore wider application of the protocol, we explored the dithiolanes containing different protecting groups. In all the cases reactions worked smoothly to afford the corresponding products $(\mathbf{2 z}, \mathbf{2 a a - 2 a c})$ in good yields. It is known that $\pi$-bond reacts with thyil radical or it gets oxidized under oxidative photoredox conditions. Gratifyingly, different functional groups were well tolerated under the reaction conditions. Further, we explored the substrate scope of dithiolanes for the sulfoxidation reaction as a proof of concept and the corresponding sulfoxidation products (3a-3d) were obtained in very good yields (up to $85 \%$ ).

Next, we switched our attention to explore the other cyclic thioacetals such as 1-3-dithianes. To begin with we subjected phenyl-1,3-dithiane 4a under optimized reaction containing DMAP as a base. To our great surprise, we obtained the corresponding sulfoxide product $\mathbf{6 a}$ in excellent yield $(90 \%$, Scheme 3) instead of the anticipated rearrangement product $\mathbf{5 a}$ and this observation found to be reproducible. Based on the literature, we surmise that most probably due to the extra stability arising in the oxidized 1,3-dithiane species (6membered ring, $\sigma-\sigma^{*}-3 e-2$ centered interaction) sulfoxide $\mathbf{6 a}$ was obtained. While, this phenomenon is believed to be absent in the oxidized 1,3-dithiolane species (5-membered ring). ${ }^{20}$

Scheme 3. Substrate scope for rearrangement and sulfoxidation of dithiane ${ }^{a}$

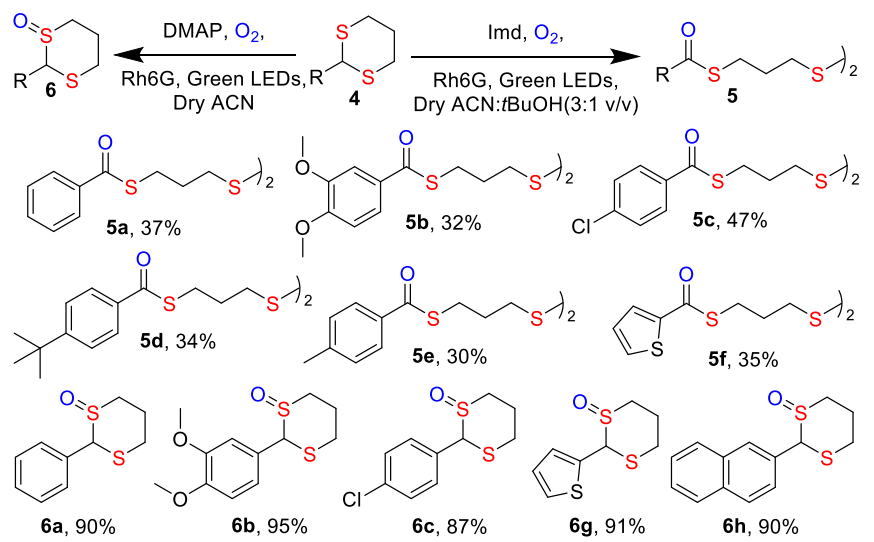

${ }^{\mathrm{a}}$ General reaction conditions: 1a $(0.2 \mathrm{mmol})$, DMAP $(0.4 \mathrm{mmol})$, Imidazole (1 mmol), Solvent $(2 \mathrm{ml})$, Green LEDs $(15 \mathrm{~W})$, under $\mathrm{O}_{2}, 24 \mathrm{~h} . *$ reaction of $4 \mathbf{a}$ at elevated temperature $\left(50^{\circ} \mathrm{C}\right)$ did not work to offer 5 a.

In order to obtain the rearrangement product disulfide-linkeddithioester, we screened different solvents, catalysts and bases systematically (see ESI 2.6, 2.7, 2.8). Based on the exhaustive screening dithiane $\mathbf{4 a}$, Rh6G as photocatalyst (2 mol\%), imidazole, in anhydrous $\mathrm{ACN}$ and $t$ - $\mathrm{BuOH}(3: 1, \mathrm{v} / \mathrm{v})$ proved to be optimum reaction condition to obtain 5a (ESI-page 2.7). Surprisingly, our attempts to increase the yield of 5a by elevating the temperature of the reaction mixture $\left(50^{\circ} \mathrm{C}\right) \mathrm{did}$ not afford the desired product even in trace. Also, based on the exhaustive screening of solvents and bases (ESI- 2.8, 2.9), 4a, DMAP, Rh6G ( $2 \mathrm{~mol} \%$ ) in dry $\mathrm{ACN}$, under $\mathrm{O}_{2}$ atmosphere and green LEDs proved to be optimum reaction condition to obtain 
6a. With these optimized reaction conditions in hand, we have screened different substrates. However, unfortunately products 5 were obtained in moderate yields in all cases ranging from electron rich to electron deactivated and heterocyclic molecules (5a, 5b, 5c, 5d, 5e, 5f). While, different substrates $(4 \mathbf{a}, 4 \mathbf{b}, 4 \mathbf{c}, 4 \mathbf{g}, \mathbf{4 h})$ under the optimized reaction condition reacted smoothly to afford the corresponding sulfoxidation products $(\mathbf{6 a}, \mathbf{6 b}, \mathbf{6 c}, \mathbf{6 g}, \mathbf{6 h})$ in excellent yields (Scheme-3, up to $95 \%$ ). We observed that dithianes favoured the sulfoxide products over the disulfide-linked-dithioesters products $\mathbf{5}$. In contrast, interestingly the dithiolanes afforded the disulfidelinked-dithioesters $\mathbf{2}$ in excellent yields. This is probably due to the relative stability of oxidized form of 6-membered cyclicthioacetals and slower ring opening.

\section{Scheme 4. Control Experiments}

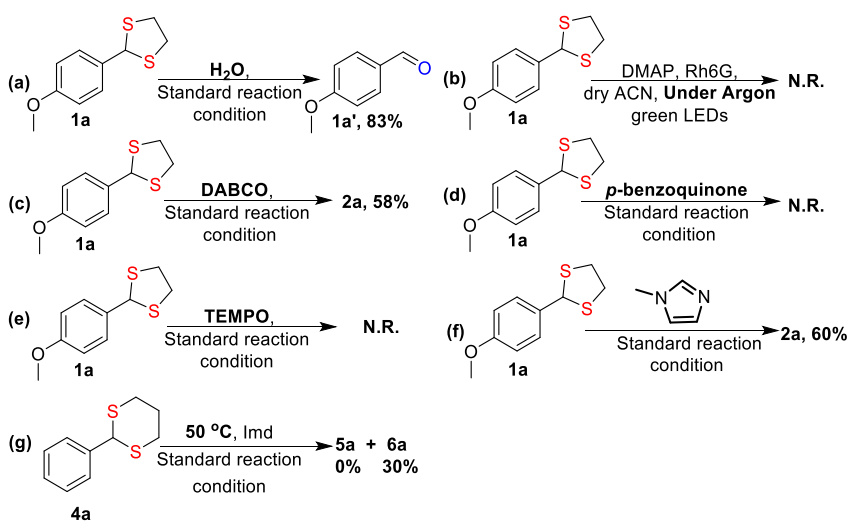

In order to have insight into the mechanism, we planned to carry out few control experiments systematically. To begin with the reaction of $\mathbf{1}$ a under optimized reaction condition but with the addition of water (10 equiv.) facilitated the deprotection to afford the corresponding aldehydes 1a' exclusively (Scheme 4a). While the reaction of $\mathbf{1 a}$ under inert atmospheric condition (absence of oxygen) did not work. This result indicated that molecular oxygen is essential for the desired transformation (Scheme $4 \mathrm{~b}$ ). In order to verify the role singlet oxygen if any, we planned to perform the reaction using singlet oxygen quencher such as DABCO. ${ }^{21}$

The reaction of $\mathbf{1 a}$ in presence of DABCO (2 equiv.) under the optimized condition afforded the desired product $\mathbf{2 a}$ in moderate yield (Scheme 4c). This result indicated that probably the singlet oxygen may not be involved in the reaction pathway. To validate the involvement of oxygen superoxide radical anion $\left(\mathrm{O}_{2}{ }^{-}-\right)$in the reaction pathway, we carried out the reaction of $1 \mathrm{a}$ in presence of a superoxide radical anion quencher $p$-benzoquinone (Scheme $4 \mathrm{~d}$ ). It is interesting to note that reaction did not proceed under the reaction condition thus strongly supporting the formation and involvement of oxygen superoxide radical anion $\left(\mathbf{O}_{2}{ }^{-}-\right){ }^{22}$ The reaction did not work in presence of TEMPO confirming the radical pathway during course of the reaction (Scheme 4e). During optimization we had observed that reaction of $1 \mathbf{a}$ in presence of imidazole furnished the corresponding sulfoxide $\mathbf{3 a}$ as a major product (Table 1, entry 10). In this regard, in order to understand the role of hydrogen bonding if any, we carried out the reaction of $1 \mathrm{a}$ in presence of $N$-methyl imidazole (instead of Imd) (Scheme $4 \mathrm{f}$ ). It is very interesting to note that we obtained the desired product $2 \mathbf{a}$ in $60 \%$ yield instead of sulfoxide $\mathbf{3 a}$. This result clearly supported the possible role of hydrogen bonding in the reaction mechanism (Scheme 4f). Further, we had observed that the reaction of $\mathbf{4 a}$ under elevated reaction temperature $\left(50^{\circ} \mathrm{C}\right)$ under the optimum reaction conditions did not afford the expected product $\mathbf{5 a}$ instead it gave $\mathbf{6 a}$ albeit in low yield (Scheme 4g). We surmise that possibly the weak hydrogen bonding might have been disrupted under the higher reaction temperature thus further supporting the role of hydrogen bonding to drive the reaction.

\section{Scheme 5. Plausible reaction mechanism}

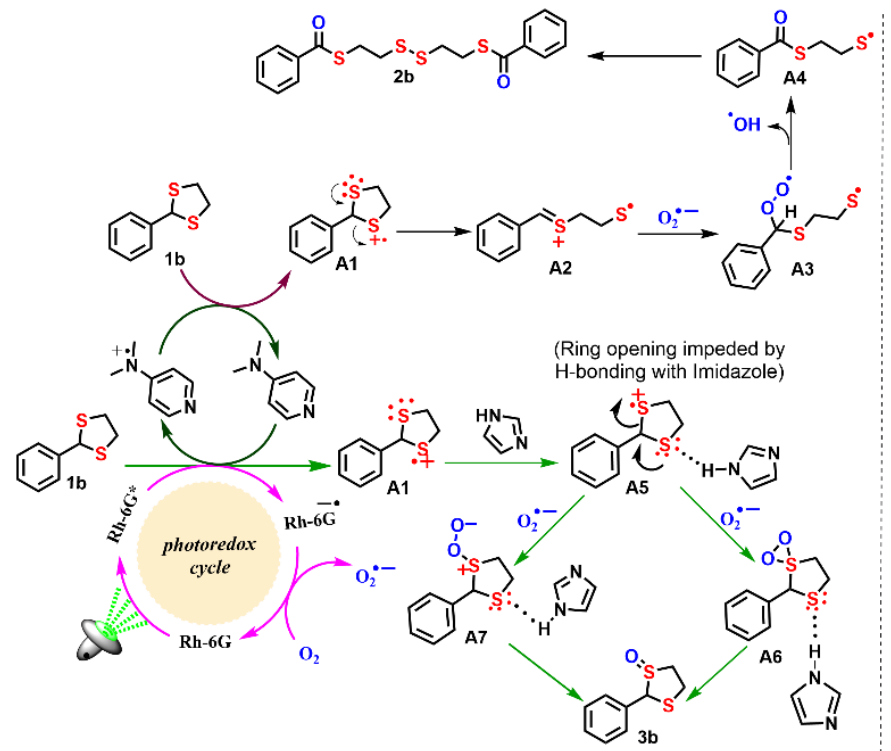

(A) Plausible mechanism to access disulphide-linked-disulphide (2a) and sulfoxide (3a) from dithiolane (1b)

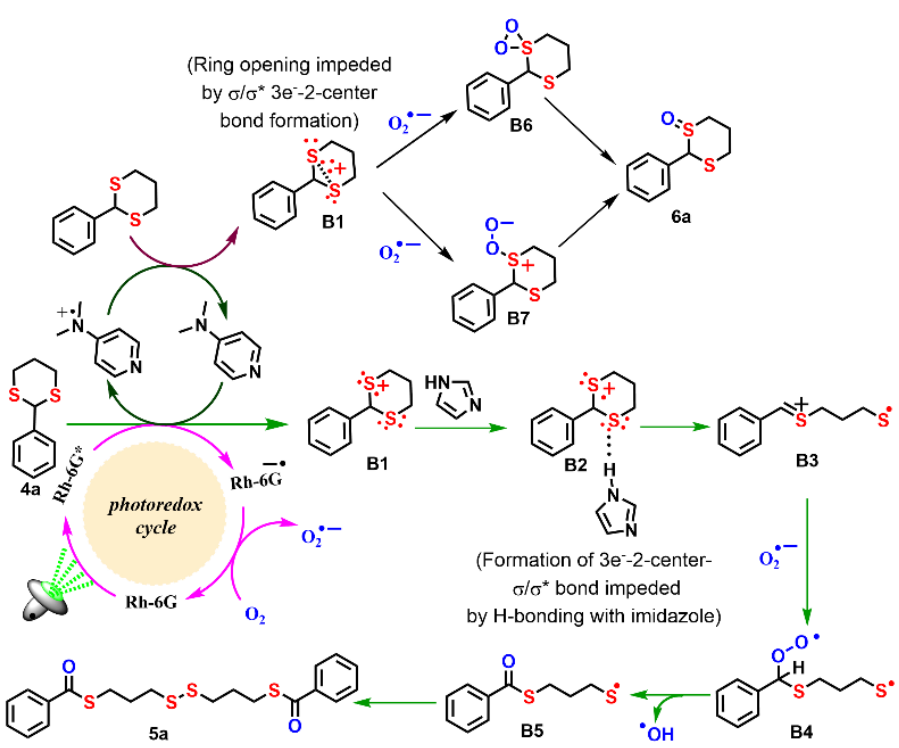

(B) Plausible mechanism to access disulphide-linked-disulphide (5a) and sulfoxide (6a) from dithiane (4a) 
Based on several control experiments, Stern-Volmer plot, cyclic voltammetry studies and previous literature reports, ${ }^{14}$ the plausible reaction pathways have been outlined (see Scheme 5A/B, see ESI for details). Based on CV experiments all the substrates have redox potential $[1 \mathbf{a}(+1.27 \mathrm{~V}), \mathbf{4 a}$ $(+1.15 \mathrm{~V})$, DMAP $(+1.27 \mathrm{~V})$, Imd $(+1.12 \mathrm{~V}) \mathrm{vs} \mathrm{Ag} / \mathrm{AgCl}]$ in the range of Rh6G $(+1.39 \mathrm{~V})^{23}$ (see ESI 6.2 A-E). Further the Stern-Volmer plot revealed that the SET is much more favourable for DMAP in comparison to $\mathbf{1 a} / \mathbf{4 a}$. While the SET is more favourable for $\mathbf{1 a} / \mathbf{4 a}$ with respect to Imd (see ESI 6.1A). Based on these experimental observations, we believe that the photocatalyst (Rh6G) upon irradiation gets excited $\left(\mathrm{Rh}_{6} *\right)(+1.39 \mathrm{~V} \text { vs SCE})^{23}$ and this would in turn takes the single electron from DMAP $(+1.27 \mathrm{~V})$ to generate the corresponding oxidized species DMAP ${ }^{\bullet+}$ and $\mathrm{Rh}^{\circ} \mathrm{G}^{\bullet-}$ (see Scheme 5a). The oxidized species DMAP ${ }^{+}$would further undergo one more SET with $\mathbf{1 b}(+1.26 \mathrm{~V})$ to afford $\mathbf{A} \mathbf{1}$ thus regenerating DMAP. The unstable oxidized species A1 collapses to generate the transient radical cation intermediate A2. This would further react with superoxide radical anion $\left(\mathbf{O}_{2}\right.$ - ) to generate the intermediate $\mathbf{A 3}$ that would eventually collapse to form the reactive radical intermediate A4. This would dimerize to afford the final product $\mathbf{2 b}$ (see Scheme 5A). While, in presence of imidazole (Imd), the SET directly happens with $\mathbf{1 b}$ instead of Imd since the quenching efficiency of $\mathbf{1 b}$ is more than Imd to generate the intermediate A1. This intermediate form a favourable hydrogen bonding with Imd to form an intermediate $\mathbf{A 5}$ that reacts with the superoxide radical $\left(\mathbf{O}_{2}{ }^{-}{ }^{-}\right)$to generate the sulfoxide product $\mathbf{3 b}$ either via $\mathbf{A 6}$ or A7 intermediate. $^{24}$ In presence of Imd, 6-membered cyclic thioacetal (dithiane, 4a) generates an intermediate B1 after SET with Rh6G* since the quenching efficiency of $\mathbf{4 a}$ is more than Imd. This intermediate is believed to be stabilized by the $3 \mathrm{e}-2$ center $\sigma-\sigma^{*}$ bond formation between two sulfur atoms in a six membered cycle reported by the Asmus et al. ${ }^{20}$ However, imidazole (Imd) having an intrinsic property of making hydrogen bonding may disturb this $3 \mathrm{e}-2$ center $\sigma-\sigma^{*}$ interaction to form an intermediate B2. This would in turn destabilize the ring to open up to generate a radical cation intermediate $\mathbf{B 3}$. Further, this intermediate would be trapped by superoxide radical anion $\left(\mathbf{O}_{2}{ }^{-}\right)$to form an intermediate $\mathbf{B} 4$. This reactive and unstable intermediate would collapse to form a radical intermediate B5. Finally, this would undergo dimerization to furnish the desired product 5a. While in presence of DMAP the intermediate B1 forms through a relay of two SET events. Stabilized intermediate B1 (by the $3 \mathrm{e}-2$ center $\sigma-\sigma^{*}$ interaction) will stay intact in presence of DMAP due to the absence of hydrogen bonding thus making it to resistant for ring opening. The intermediate $\mathbf{B} 1$ eventually reacts with superoxide radical anion $\left(\mathbf{O}_{2}{ }^{--}\right)$to furnish the desired sulfoxide product $\mathbf{6 a}$ via either intermediate $\mathbf{B 6}$ or B7. ${ }^{24}$

Based on plausible reaction mechanism (Scheme 5A), we predicted that in situ generated thiyl radiacal intermediate (A4) could be trapped with the suitable disulfide to synthesize the hetero-disulfide compounds. However, synthesis of heterodisulfide is quite challenging as homo-disulfide is also known to form. ${ }^{25}$ In order to extend the utility of this protocol further, we planned to explore the synthesis of hetero-disulfides (Scheme 6). We treated dithiolanes (1) with little excess of aromatic disulfides (7a-7e) under the optimized reaction conditions to afford the corresponding hetero disulfides (8aa8ie) in moderate to good yields (up to $73 \%$, Scheme 6 ). We observed that disulfides (7) containing electron-donating substituents as well as free hydroxyl group as substituent worked smoothly under the conditions while the disulfide containing electron withdrawing groups did not work.

\section{Scheme 6. Substrate scope for the disulfide trapping}

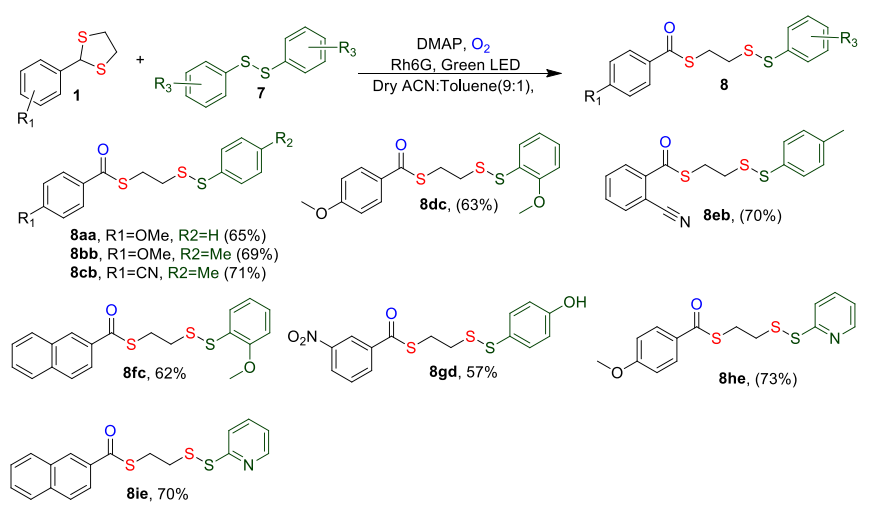

${ }^{\mathrm{a}}$ General reaction conditions: $\mathbf{1}(0.2 \mathrm{mmol}), \mathbf{7}(0.6 \mathrm{mmol})$, DMAP $(0.4 \mathrm{mmol})$, Solvent $(3 \mathrm{~mL})$, Green LEDs $(15 \mathrm{~W})$, under $\mathrm{O}_{2}$.

We also observed that the aliphatic disulfides were ineffective under the conditions to furnish the corresponding heterodisulfide compounds. For the practicality of the protocol, we further demonstrated the gram scale reaction of 1a under the standard conditions to afford the desired product $\mathbf{2 a}(62 \%$, see ESI, 4.5).

\section{Conclusions}

In conclusion we have developed a visible light mediated and base dependent rearrangement reaction of dithiolane as well as dithiane to afford the disulfide-linked-dithioesters and sulfoxide via photoredox catalysis under room temperature. A wide range of dithiolanes and dithianes reacted smoothly under the reaction conditions to afford a variety of sulfoxides and disulfide-linked-dithioesters. We have also demonstrated the practicality of the protocol by synthesizing the suitable heterodisulfides via trapping the thiyl radical with aryldisulfides. We studied the unusual reactivity of dithiolanes and dithianes by manipulating the bases with and without hydrogen bonding capability under photooxidative conditions for the very first time. This one pot protocol does not rely on external oxidizing reagents or metal reagents and works well under the mild reaction condition. The method gives an access to very useful molecules with two different functional groups in a single step starting from easily synthesizable dithiolanes and dithanes. The protocol has been proven to be scalable on gram quantity. The observed unique reactivity of thioacetals may open up the new horizon for synthesizing different useful molecules by exploring reactions with different nucleophiles. 


\section{Author Contributions}

\section{Conflicts of interest}

There are no conflicts to declare".

\section{Acknowledgements}

R. G. B. thanks DST-SERB (CRG/2019/005753), Govt. of India for the generous research grant. Authors also thank IISER Pune for the financial assistance. P. D. D. thank CSIR, New Delhi and A. S. T. thanks DST-SERB New Delhi, Government of India for providing fellowship.

\section{Notes and references}

1. Kaiser, D.; Klose, I.; Oost, R.; Neuhaus, J.; Maulide, N. Bond-Forming and -Breaking Reactions at Sulfur(IV): Sulfoxides, Sulfonium Salts, Sulfur Ylides, and Sulfinate Salts. Chem. Rev. 2019, 119, 8701-8780.

2. (a) Corey, E. J.; D. Seebach, D. Carbanions of 1,3Dithianes. Reagents for C-C Bond Formation by Nucleophilic Displacement and Carbonyl Addition. Angew. Chem. Int. Ed. Engl. 1965, 4, 1077; Angew. Chem. 1965, 77, 1136. (b)Corey, E. J.; D. Seebach, D. Generation and synthetic applications of 2-lithio1,3-dithianes. J. Org. Chem. 1975, 40, 231; (c)Seebach, D. Methods of Reactivity Umpolung. Angew. Chem. Int. Ed. Engl. 1979, 18, 239; Angew. Chem. 1979, 91, 259.

3. (a) Herbst, D. A.; Townsend, C. A.; Maier, T. The Architectures of Iterative Type I PKS and FAS. Nat. Prod. Rep., 2018, 35, 1046-1069.

4. Franke, J.; Hertweck, C.; Biomimetic Thioesters as Probes for Enzymatic Assembly Lines: Synthesis, Applications, and Challenges, Cell Chem. Bio. 2016, 23, 1179-1192.

5. (a) Novel thiophosphoramidites, Bleicher, K.; Duschmale, J.; Brigitte, M.; Henrik, D.; Hansen, F.; Koch, T.; Li, M.; Schaeublin, A.; Sue, Xi.; Wu, Y. PTC/EP2018/086457, 27 June 2019. (b) Yang, X.; Sierant, M.; Janicka, M.; Peczek, L.; Martinez, C.; Hassell, T.; Li, N.; Li, X.; Wang, T.; Nawrot, B. Gene Silencing Activity of siRNA Molecules Containing Phosphorodithioate Substitutions, ACS Chem. Biol., 2012, 7, 1214-1220. (c) Wiesler, W. T.; Caruthers, M. H. Synthesis of Phosphorodithioate DNA via Sulfur-Linked, Base-Labile Protecting Groups. J. Org. Chem. 1996, 61, 4272-4281.

6. (a) Kazemia, M.; Shiri, L. Thioesters synthesis: recent adventures in the esterification of thiols. Journal of Sulfur Chemistry, 2015, 36, 613-623. (b) Zheng, T.-C.; Burkart, M.; Richardson, D. E. A general and mild synthesis of thioesters and thiols from halides. Tetrahedron lett., 1999, 40, 603-606.
7. (a) Li, C. F.; Xiao, W. J.; Alper, H. PalladiumCatalyzed Ring-Opening Thiocarbonylation of Vinylcyclopropanes with Thiols and Carbon Monoxide. J. Org. Chem. 2009, 74, 888-890; (b) Cao, H.; McNamee, L.; Alper, H. PalladiumCatalyzed Thiocarbonylation of Iodoarenes with Thiols in Phosphonium Salt Ionic Liquids. J. Org. Chem., 2008, 73, 3530-3534; (c) Burhardt, M. N.; Ahlburg, A.; Skrydstrup, T. Palladium-Catalyzed Thiocarbonylation of Aryl, Vinyl, and Benzyl Bromides. J. Org. Chem., 2014, 79, 11830-11840. (d) Kang, B.; Hong, S. K. Photoredox mediated nickel catalyzed $\mathrm{C}\left(\mathrm{sp}^{3}\right)-\mathrm{H}$ thiocarbonylation of ethers. Chem. Sci. 2017, 8, 6613-6618.

8. (a) Yi, C.; Huang, Y.; Lee, C. Synthesis of thioesters through copper-catalyzed coupling of aldehydes with thiols in water. Green Chem. 2013, 15, 2476. (b) Chung, J.; Seo, U. R.; Chun, S.; Chung, Y. K. Poly(3,4-dimethyl-5-vinylthiazolium)/DBU-

Catalyzed Thioesterification of Aldehydes with Thiols. ChemCatChem. 2016, 8, 318-321. (b) Xie, S.; Su, L.; Mo, M.; Zhou, W.; Zhou, Y.; Dong, J. CuCatalyzed Oxidative Thioesterification of Aroylhydrazides with Disulfides. J. Org. Chem. 2021, 86, 739-749. (c) Shi, Y.; Liu, X.; Cao, H.; Bie, F.; Han, Y.; Yan, P.; Szostak, R.; Szostak, M.; Liu, C. Conversion of esters to thioesters under mild conditions, Org. Biomol. Chem. 2021, 19, 29912996.

9. (a) Thorton, J. M. Disulphide Bridges in Globular Proteins. J. Mol. Biol. 1981, 151, 261-287; (b) Hogg, P. J. Disulfide bonds as switches for protein function. Trends Biochem. Sci. 2003, 28, 210-214; (c) Saunders, A. J.; Young, G. B.; Pielak, G. J. Polarity of disulfide bonds. Protein Sci. 1993, 2, 1183-1184.

10. (a) Echelman, D. J.; Lee, A. Q.; Fernández, J. M. Mechanical forces regulate the reactivity of a thioester bond in a bacterial adhesion. J. Biol. Chem. 2017, 292, 8988-8997; (b) Folikumah, M. Y.; Behl, M.; Lendlein, A. Thiol-Thioester Exchange Reactions in Precursors Enable pH-Triggered Hydrogel Formation. Biomacromolecules, 2021, 22, 1875-1884; (c) Xuan, W.; Collins, D.; Koh, M.; Shao, S.; Yao, A.; Xiao, H.; Garner, P. P.; Schultz, P.G. Site-specific Incorporation of a Thioester Containing Amino Acid into Proteins. ACS Chem. Biol. 2018, 13, 578-581; (d) Schuler, B.; Pannell, L. K. Specific Labeling of Polypeptides at AminoTerminal Cysteine Residues Using Cy5-benzyl Thioester. Bioconjug. Chem. 2002, 13, 1039-1043; (e) Sletten E. M.; Bertozzi, C. R. Bioorthogonal Chemistry: Fishing for Selectivity in a Sea of Functionality. Angew. Chem. Int. Ed. 2009, 48, 6974-6998; (f) Rinalducci, S.; Murgiano. L.; Zolla, L. Redox proteomics: basic principles and future perspectives for the detection of protein oxidation in plants. J. of Exp. Bot. 2008, 59, 3781-3801. (g) Trang Le, N. T.; Quynh Nguyen, T. N.; Cao, V. D.; 
Hoang, D. T.; Ngo, V. C.; Hoang Thi, T. T. Recent Progress and Advances of Multi-Stimuli-Responsive Dendrimers in Drug Delivery for Cancer Treatment. Pharmaceutics, 2019, 11, 591; (h) Altinbasak, I.; Arslan, M.; Sanyal, R.; Sanyal, A. Pyridyl disulfide-based thiol-disulfide exchange reaction: shaping the design of redox-responsive polymeric materials, Polym. Chem., 2020, 11, 7603-7624. (i) Winther, J. R.; Thorpe, C. Quantification of thiols and disulfides, 2014, 1840, 838-846. (i) Konievab, O.; Wagner, A. Developments and recent advancements in the field of endogenous amino acid selective bond forming reactions for bioconjugation. Chem. Soc. Rev, 2015, 44, 5495. (J) Trivedi, M. V.; Laurence, J. S.; Siahaan, T. J. The Role of Thiol and Disulfide on Protein Stability. Curr. Protein and Pept. Sci. 2009, 10, 614-625. (k) Stasińskaa, A. R.; Putaj, P.; Chmielewskia, M. K. Disulfide bridge as a linker in nucleic acids, bioconjugation. Part II: A summary of practical applications. Bioorg. Chem. 2020, 95, 103518.

11. D. de la Fuente-Herreruela, D.; Monnappa, A. K.; Muñoz-Úbeda, M.; Morallón-Piña, A.; Enciso, E.; Sánchez, L.; Giusti, F.; Natale, P.; López-Montero, I. Lipid-peptide bioconjugation through pyridyl disulfde reaction chemistry and its application in cell targeting and drug delivery. J. Nanobiotechnol, 2019, 17, 77.

12. (a) Crisenza, G.; Melchiorre, P. Chemistry Glows Green with Photoredox Catalysis. Nat. Commun. 2020, 11, 803. (b) McAtee, R. C., McClain, E. J.; Stephenson, C. R. J. Illuminating Photoredox Catalysis. Trends Chem. 2019, 1, 111-125. (c) Shaw, M. H.; Twilton, J.; MacMillan, D. W. C. Photoredox Catalysis in Organic Chemistry. J. Org. Chem. 2016, 81, 6898-6926. (d) Reischauer, S.; Pieber, B. Emerging Concepts in Photocatalytic Organic Synthesis. iScience, 2021, 24, 102209. (e) Wimmer, A.; and König, B. Photocatalytic Formation of Carbon-Sulfur Bonds. Beilstein J. Org. Chem. 2018, 14, 54-83. (f) Teders M.; Henkel, C.; Anhäuser, L.; Strieth-Kalthoff, F.; Gómez-Suárez, A.;Kleinmans, R.; Kahnt, A.; Rentmeister, A.; Guldi, D.; Glorius, F. The Energy-transfer-enabled Biocompatible Disulfide-ene Reaction. Nat. Chem. 2018, 10, 981988.

13. (a)Lanzi, M.; Merad, J.; Boyarskaya, D. V.; Maestri, G.; Allain, C.; Masson, G. Visible-Light-Triggered $\mathrm{C}-\mathrm{C}$ and $\mathrm{C}-\mathrm{N}$ Bond Formation by $\mathrm{C}-\mathrm{S}$ Bond Cleavage of Benzylic Thioethers. Org. Lett., 2018, 20, 5247-5250. (b) Hong, B.; Aganda, K. C. C.; Lee, A. Oxidative $\mathrm{C}-\mathrm{S}$ Bond Cleavage of Benzyl Thiols Enabled by VisibleLight-Mediated Silver(II) Complexes. Org. Lett. 2020, 22, 4395-4399. (c) Krumb, M.; Lucas, T.; Opatz, T. Visible Light Enables Aerobic Iodine Catalyzed Glycosylation. Eur. J. Org. Chem. 2019, 28, 4517-4521.

14. (a) Dharpure, P. D.; Bhowmic, A.; Warghude, P. K.; Bhat, R. G. Tetrahedron Lett., 2020, 61, 151407. (b) Oksdath-Mansilla, G.; Hajj, V.; Andrada, D. M.; Argüello, J. E.; Bonin, J.; Robert, J. M.; Peñeńory,
A. B. Photoremoval of Protecting Groups: Mechanistic Aspects of 1,3-Dithiane Conversion to a Carbonyl Group. J. Org. Chem. 2015, 80, 2733. (c) Matthias Krumb, Lisa Marie Kammer, Robert Forster, Caroline Grundke, Prof. Dr. Till Opatz. Visible-Light-Induced Cleavage of $\mathrm{C}-\mathrm{S}$ Bonds in Thioacetals and Thioketals with Iodine as a Photocatalyst. ChemPhotoChem. 2020, 4, 101-104.

15. (a) Pandey, B.; Smita Y. Bal, Khire, U. R. Photosensitized Electron Transfer Oxidation of Zsubstituted 1,3-dithiolane to 1,3-dithiolane-1-oxide. Tetrahedron Lett., 1989, 30, 4007-4008. (b) Pandey, B.; Smita Y. Bal, Uday R. Khire, Rao, A. T. Efficient Synthetic Methodology for 1,3-Dithiolane 1 -Oxides via Singlet Oxidation of 1,3-Dithiolanes. J. Chem. Soc. Perkin Trans, 1. 1990, 11, 3217. (c) Gu, X.; Li, X.; Chai, Y.; Yang, Q.; Li, P.; Yao, Y. A Simple Metal-free Catalytic Sulfoxidation Under Visible Light and Air. Green Chem. 2013, 15, 357-361. (d) Zhang, S.; Li, G.; Li, L.; Deng, X.; Zhao, G.; Cui, X.; Tang, Z. Alloxan-Catalyzed Biomimetic Oxidations with Hydrogen Peroxide or Molecular Oxygen. ACS Catal. 2020, 10, 245-252. (e) Clennan, E. L.; Liao, C. Synthesis, Characterization, Photophysics and Photochemistry of Pyrylogen Electron Transfer Sensitizers. Photochem. and Photobio. 2014, 90, 344-357.

16. Valiulin, R. A.; Kottani, R.; Kutateladze, A. G.; When Ethyl Is Infinitely Different from Methyl: Double Addition of Lithiated Dithianes to Aromatic Carboxylates Revisited. J. Org. Chem. 2006, 71, 5047.

17. Vale, J. R.; Rimpiläinen, T.; Sievänen, E.; Rissanen, K.; Afonso, C. A. M.; Candeias, N. R. Pot-Economy Autooxidative Condensation of 2-Aryl-2-lithio-1,3dithianes. J. Org. Chem. 2018, 83, 1948-1958.

18. Abidi, N.; Schmink, J. R. Synthesis of Disubstituted Dithioethers: tert-Butoxide Promoted Elimination/Ring Opening of 1,3-Dithianes Followed by Palladium-Catalyzed $\mathrm{C}-\mathrm{S}$ Bond Formation. J. Org. Chem. 2015, 80, 4123-4131.

19. Formation of a very few disulfide-linked-dithioesters have been reported as unanticipated side products during the deprotection of thioacetals in presence of stoichiometric amount of DDQ. However, the systematic study and the wider substrate scope for the same has not been explored till date. See ref. (a) Tanemura, K., Dohya, H., Imamura, M., Suzuki, T., Horaguchi, T. Deprotection of 1, 3-Dithianes by 2, 3Dichloro-5,6-dicyano-p-benzoquinone (DDQ). Chemistry Lett., 1994, 965-968; (b) Ichige, T.; Miyake, A.; Kanoh, N.; Nakata, M. Oxidative Deprotection of 1, 3-Dithiane Group Using $\mathrm{NaClO}_{2}$ and $\mathrm{NaH}_{2} \mathrm{PO}_{4}$ in Aqueous Methanol. Synlett, 2004, 10, 1686-1690. (c) Firouzabadi, H.; Iranpoor, N. Deprotection of S,S-acetals. Sci. of Synth., 2007, 30, 505-586.

20. Asmus, K.-A. Stabilization of Oxidized Sulfur Centers in Organic Sulfides Radical Cations and Odd-Electron Sulfur-Sulfur Bonds, Accounts of Chemical Research, 1979, 12, 436-442. 
21. Guo, J.-T.; Yang, D.-C.; Guan, Z.; He, Y.-H. Chlorophyll-Catalyzed Visible-Light-Mediated Synthesis of Tetrahydroquinolines from $N, N-$ Dimethylanilines and Maleimides. J. Org. Chem. 2017, 82, 1888-1894. (DABCO- singlet oxygen quencher ref.)

22. Pelaez, M.; Falaras, P.; Likodimos, V.; O'shea, K.; de la Cruz, A.; Dunlop, P. S. M.; Byrne, J. A.; Dionysiou, D. D. Use of Selected Scavengers for the Determination of NF-TiO2 Reactive Oxygen Species During the Degradation of Microcystin LR under Visible Light Irradiatio. J. Mol. Catal. A Chem. 2016, 15, 183-189.

23. (a)Dietrich, A.; Buschmann, V.; Müller, C.; Sauer, M. Fluorescence resonance energy transfer (FRET) and competing processes in donor-acceptor substituted DNA strands: a comparative study of ensemble and single-molecule data. Rev. Mol. Biotechno., 2002, 82, 211-231. (b) Sören Doose, S.; Neuweiler, H,; Sauer, M. Fluorescence Quenching by Photoinduced Electron Transfer: A Reporter for Conformational Dynamics of Macromolecules. ChemPhysChem, 2009, 10, 13891398.

24. Miller, B. L.; Williams, T. D.; Schöneich, C. Mechanism of Sulfoxide Formation through Reaction of Sulfur Radical Cation Complexes with Superoxide or Hydroxide Ion in Oxygenated Aqueous Solution. J. Am. Chem. Soc. 1996, 118, 11014-11025.

25. (a) Hunter, R.; Caira, M.; Stellenboom, N. Inexpensive, One-Pot Synthesis of Unsymmetrical Disulfides Using 1-Chlorobenzotriazole. J. Org. Chem. 2006, 71, 8268-8271. (b) Spiliopoulou, N.; Kokotos, C. G. Photochemical metal-free aerobic oxidation of thiols to disulfides. Green Chem., 2021, 23, 546-551. (C) Dethe, D. H.; Srivastava, A.; Dherange, B. D.; Kumar, B. V. Unsymmetrical Disulfide Synthesis through Photoredox Catalysis. Adv. Synth. Catal, 2018, 360, 3020-3025. 
Table of Contents

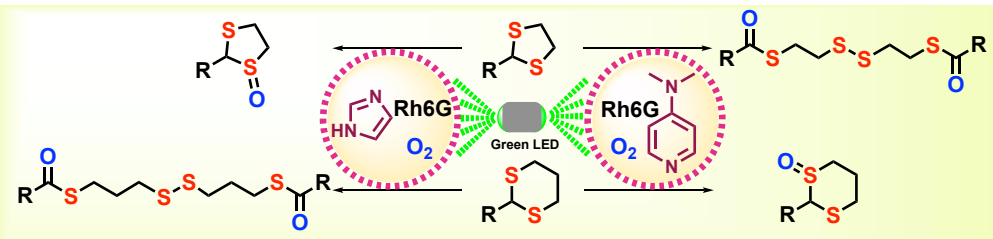

- Selective thioacetal rearrangement - Regeoselective oxidation Base dependent product formation - Metal free and ambient protocol Functional group tolerance Total of 53 examples and scalable 